\title{
Adsorbentes no-convencionales, alternativas sustentables para el tratamiento de aguas residuales ${ }^{*}$
}

\author{
María Guadalupe Valladares-Cisneros* \\ Cintya Valerio Cárdenas ${ }^{* * *}$ \\ Patricia de la Cruz Burelo*** \\ Rosa María Melgoza Alemán ${ }^{* * * * *}$
}

Recibido: 10/07/2016 • Aceptado: 21/12/2016

DOI: 10.22395/rium.v16n31a3

\begin{abstract}
Resumen
Se presenta una revisión sobre los adsorbentes no-convencionales utilizados para la remoción de contaminantes en el agua. Se describirán brevemente los adsorbentes convencionales para distinguir a los no-convencionales. Los adsorbentes convencionales son aquellos materiales naturales o sintéticos los cuales, para ser empleados, deben recibir un tratamiento para activarse, como el carbono, las arcillas, las membranas etc.; después de usarse estos materiales tienen la cualidad de poder ser regenerados. Los adsorbentes no-convencionales son materiales alternos (biopolímeros o partes de plantas); no necesariamente deben recibir un tratamiento previo para activarse; sin embargo, su activación mejora su capacidad de adsorción. Las fuentes estudiadas para la obtención de materiales empleados como adsorbentes no-convencionales han sido los residuos agroindustriales y de la industria alimenticia y especies vegetales cuya aplicación es restringida o no tienen aplicación. Han sido diferentes los estudios que demuestran que el empleo de materiales naturales como adsorbentes no-convencionales permite reducir la carga orgánica de un efluente contaminado con metales, colorantes, pesticidas y algunos otros compuestos orgánicos denominados emergentes. Las áreas de estudio para los materiales no-convencionales surgen como una necesidad en el diseño de procesos sustentables para el tratamiento de aguas residuales, por lo que su estudio y revisión son de especial interés para profundizar y proponer alternativas acordes con las necesidades existentes.
\end{abstract}

Palabras clave: adsorbente convencional; adsorbente no-convencional; aguas residuales; materiales naturales; tratamiento sustentable.

* Artículo escrito en el marco del proyecto de investigación Estudio de tejidos vegetales como adsorbentes naturales para la remoción de colorantes en medio líquido desarrollado en el año 2016 con recursos del Programa de Fortalecimiento de la Calidad en Instituciones Educativas (Profocies-2015) de México. Entidades participantes: Universidad de la Chontalpa y Universidad Autónoma del Estado de Morelos.

** PhD. Facultad de Ciencias Químicas e Ingeniería. Universidad Autónoma del Estado de Morelos. Avenida Universidad 1001, Col. Chamilpa, Cuernavaca Mor. C.P. 62209, México. Tel/Fax. 777-329- 70-00, Ext. 3746. dra.mg.valladares.cisneros@gmail.com

*** Ingeniera Química. División de Ciencias Básicas e Ingeniería. Universidad Popular de la Chontalpa. Carretera Cárdenas-Huimanguillo km 2. R / a. Paso y playa s / n. H. Cárdenas, Tabasco, México. cintyavaca@gmail.com

**** PhD. Maestría en Química Sustentable. Universidad Popular de la Chontalpa. Carretera Cárdenas-Huimanguillo km 2. R/a. Paso y playa s/n. H. Cárdenas, Tabasco, México. patyburelo@hotmail.com

${ }^{* * * * *}$ Ph.D. Facultad de Ciencias Químicas e Ingeniería. Universidad Autónoma del Estado de Morelos. Avenida Universidad 1001, Col. Chamilpa, Cuernavaca Mor. C. P. 62209, México.rmelgoza@uaem.mx 


\title{
Non-conventional absorbers: sustainable alternatives for wastewater treatment
}

\begin{abstract}
This article shows a revision of the non-conventional absorbers employed for the removal of water pollutants. A brief description is made on the conventional absorbers in order to distinguish them from the non-conventional ones. Conventional absorbers are those natural or synthetic materials which employment is contingent with a treatment process so that they can be activated, such as carbon, clays, membranes, etc. After using these materials, they are able to be regenerated. Non-conventional absorbers are alternate materials (biopolymers or parts of plants); they should not necessarily be subject to a previous treatment to be activated; however, activation improves their absorption capacity. Sources studied for obtaining the materials employed as non-conventional absorbers include agroindustrial wastes, food industry wastes, and plant species which application is restricted or has no application at all. Different studies have shown that the employment of natural materials as non-conventional absorbers allows minimizing the organic discharge of a river contaminated with metals, dyes, pesticides, and other organic compounds known as emerging compounds. The fields of study for non-conventional materials emerge as a need for the design of sustainable process for the wastewater treatment; for this reason, the study and revision of non-conventional materials are of special interest to go deeper and propose alternative according to the existing needs. Keywords: non-conventional absorbers; sustainable treatment; natural materials; wastewater; conventional absorber.
\end{abstract}

\section{Adsorventes não convencionais, alternativas sustentáveis para o tratamento de águas residuais}

\begin{abstract}
Resumo
Apresenta-se uma revisão sobre os adsorventes não convencionais utilizados para a remoção de poluentes na água. São descritos brevemente os adsorventes convencionais para diferenciá-los dos não convencionais. Aqueles são materiais naturais ou sintéticos que, para serem empregados, devem receber um tratamento para ativar-se, como carbono, argila, membranas, entre outros. Os não convencionais são materiais alternativos (biopolímeros ou partes de plantas); não necessariamente devem receber um tratamento prévio para ativar-se, mas sua ativação melhora a capacidade de adsorção. As fontes estudadas para obter materiais empregados como adsorventes não convencionais são os resíduos agroindustriais e da indústria alimentar, bem como espécies vegetais cuja aplicação é restringida ou não têm aplicação. São diferentes os estudos que demonstram que o emprego de materiais naturais como adsorventes não convencionais permite reduzir a carga orgânica de um efluente poluído com metais, corantes, pesticidas e outros componentes orgânicos denominados emergentes. As áreas de estudo para os materiais não convencionais surgem como uma necessidade no desenho de processos sustentáveis para o tratamento de águas residuais, razão pela qual seu estudo e revisão são de especial interesse para propor alternativas concordantes com as necessidades existentes e aprofundar-se no tema.
\end{abstract}

Palavras-chave: adsorvente convencional: adsorvente não convencional; águas 


\section{INTRODUCCIÓN}

Al formar parte integral de los seres vivos el agua ha sido considerada "el líquido vital". El protoplasma celular contiene aproximadamente un $80 \%$ de agua, por lo que todos necesitamos para beber agua que sea limpia y segura [1]. Por sus propiedades fisicoquímicas, el agua puede disolver y contener sustancias químicas (inorgánicas y orgánicas); por ello es denomina apropiadamente "El solvente universal"; inclusive llega a albergar organismos microscópicos [2].

El agua presenta gran relevancia en la economía mundial, porque es indispensable en diversas actividades como la agricultura y la industria, acarreando como consecuencia una demanda, misma que se incrementa día con día y que genera grandes volúmenes de agua residual que requieren ser tratados antes de su disposición final.

Las actividades industriales y agrícolas conducen a una contaminación química del agua por metales, colorantes, plaguicidas y otros compuestos que se disponen en menor proporción. En años recientes se ha detectado en el agua la presencia de sustancias denominadas contaminantes emergentes, entre los que se contemplan fármacos principalmente de tipo endocrino y otras sustancias orgánicas para las cuales su uso se incrementa día con día, favoreciendo su acumulación. Estos contaminantes no se encuentran normados en la mayoría de los países latinoamericanos por lo que las normas actuales requieren de actualización.

Actualmente los problemas de la contaminación se han vuelto opresivos, porque tanto el crecimiento demográfico como la expansión per cápita en el consumo de materiales y energía aumentan las cantidades de desechos y residuos que se disponen al ambiente, lo que provoca que las regulaciones en el control de contaminantes, sobre todo en la calidad de agua, sean más estrictas en muchos países.

En el Programa Nacional Hídrico 2014-2018, México contempla nuevas formas de políticas hídricas y gestión del recurso hídrico (CONAGUA, 2014). El panorama del agua en México es preocupante por lo que el Gobierno de la República lo reconoce como un asunto de seguridad nacional, procurando el cuidado del agua y el saneamiento de las aguas residuales, y destaca que son metas relevantes a cumplir, enfatizando que la crisis del agua pone en riesgo el desarrollo que pudiera tener el país en los próximos años [3].

Dependiendo de su origen las aguas residuales pueden contener diversos contaminantes, muchos de los cuales han sido clasificados como contaminantes peligrosos debido a su alta toxicidad para la vida acuática y la salud humana. Ante esta situación se han desarrollado alternativas de tratamiento de aguas residuales. Diversos métodos se 
han reportado en la literatura y son empleados para la eliminación de contaminantes en solución acuosa. Las tecnologías de tratamiento incluyen como métodos: precipitación química, intercambio de iones, procedimiento de separación de membrana, degradación biológica, oxidación química, extracción con disolventes y adsorción $[4,5]$.

La adsorción es un método que ha demostrado ser prometedor para la eliminación de contaminantes en medios acuosos. En este trabajo se revisan y distinguen estudios realizados con adsorbentes no-convencionales (materiales naturales) empleados como alternativas sustentables para la remoción de metales pesados y colorantes en agua. Esta tecnología resulta sumamente atractiva por su bajo costo, facilidad, simplicidad, alta eficiencia y sobre todo por la degradabilidad de los materiales naturales.

\section{CONTAMINANTES EN AGUA}

Las aguas residuales pueden contener diferentes sustancias contaminantes en diferente concentración. Algunos autores mencionan que deben tenerse mayormente en cuenta aquellas sustancias que en concentraciones inferiores a la concentración traza causan serios problemas a la salud, como, por ejemplo los compuestos disruptores endocrinos $[6,7]$.

En general, los contaminantes orgánicos presentes en las aguas residuales incrementan la demanda bioquímica de oxígeno (DBO), así como la demanda química de oxígeno (DQO), conduciendo al agotamiento del contenido de oxígeno en el agua y dando como resultado la hipertroficación, lo que amenaza la vida acuática. Los contaminantes orgánicos en las aguas residuales principalmente son de tipo ácido tales como compuestos carboxílicos y ácidos sulfónicos y fenólicos, porque estos compuestos tienen una buena solubilidad en agua, pero limitada biodegradación [8].

Derivadas de las actividades industriales como la aeroespacial, la minería, la metalurgia, el acabado de metales, la producción de aleaciones, la industria automotriz, y la producción de pinturas, baterías, conservantes y pesticidas se producen continuamente descargas de aguas residuales con alta concentración de iones metálicos [9 - 11].

Los metales pesados a bajas concentraciones provocan efectos tóxicos para animales, plantas y seres humanos; todo ello, a través de la cadena alimenticia $[12,13]$. Metales como plomo $(\mathrm{Pb})$, cromo $(\mathrm{Cr})$, mercurio $(\mathrm{Hg})$, talio $(\mathrm{Tl})$, selenio $(\mathrm{Se})$, cinc $(\mathrm{Zn})$, arsénico (As), cadmio (Cd), cobalto (Co), cobre (Cu) y níquel (Ni) son considerados un grave problema ambiental debido a la que no son degradables. Los metales pesados se remueven del agua usualmente por precipitación, transformándolos en los hidróxidos respectivos; sin embargo, este método causa mayores problemas ambientales y de salud, por su corrosividad y reactividad. 
Una preocupación ambiental prioritaria e importante se deriva de la contaminación del agua por descargas de colorantes y tintes, debido a las actividades industriales textiles, producción de papel, cosméticos y por las industrias procesadoras de alimentos. Los colorantes son altamente visibles aun en concentraciones inferiores a las concentraciones traza, provocando problemas estéticos y ecológicos para los ecosistemas acuáticos [14-16].

La presencia de los colorantes en agua, aún en muy bajas concentraciones, es notoria y no deseable. A través de la estructura química los colorantes son clasificados como catiónicos, aniónicos y no iónico. De acuerdo con el Índice Internacional del Color (CI, por sus siglas en inglés) y por su forma de aplicación, los colorantes se clasifican como: ácidos, básicos, directos, dispersos, con brillo fluorescente, para piel, mordentes, pigmentos, reactivos, azufrados y cuba. El grupo funcional responsable de impartir el color a la molécula se denomina cromóforo (figura 1), y es considerado por el CI para clasificar a los colorantes como azoicos, nitrosos, nitro, antraquinona, triarilmetano, metino, azina, oxacina, índigo, ftalocianina, estilbeno, xanteno, acridina, quinolina, triazol y tintes de complejos metálicos.

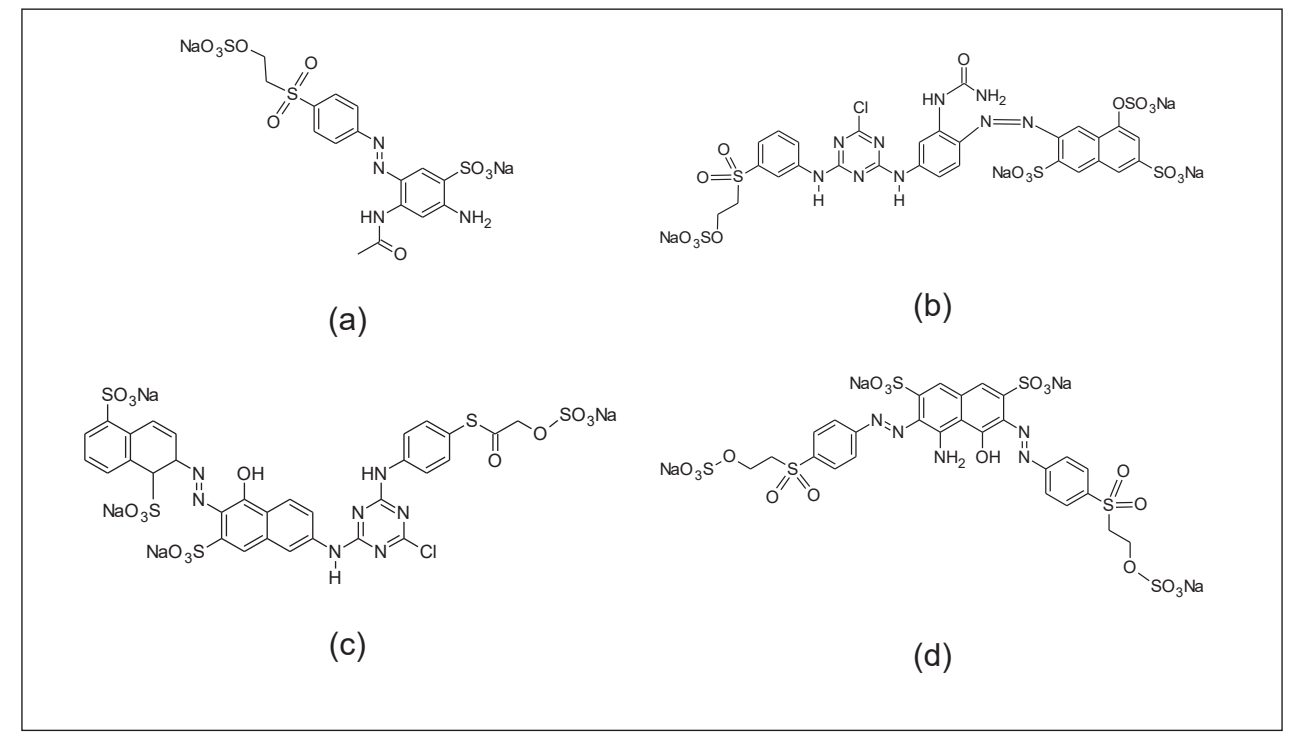

(a) Naranja reactivo 107, (b) Amarillo reactivo 145, (c) Naranja reactivo 122, (d) Negro reactivo 5. En todos los compuestos se puede apreciar al grupo R-N=N-R' al que se nombra cromóforo.

Figura 1. Estructuras químicas de algunos colorantes azoicos. Fuente: elaboración propia

La presencia de colorantes en agua disminuye la penetración de la luz solar y reduce la fotosíntesis y otras actividades biológicas propias de los organismos acuáticos 
$[17,18]$. En la fabricación de los colorantes se emplean como materias primas sustancias carcinogénicas o mutagénicas, por ejemplo, la bencidina, sustancia altamente tóxica y con la cual se sintetizan muchos de los colorantes de tipo aromático [19].

Es la industria textil la que consume altos volúmenes de agua en sus procesos de teñido, estimándose un consumo de aproximadamente $100 \mathrm{~mL} / \mathrm{Kg}$ de tela [20]. También es el principal sector que aprovecha los colorantes comercialmente existentes, consumiendo cerca del $50 \%$ de la producción anual mundial [21, 22].

La falta de legislación actualizada para la descarga de efluentes ha conducido a que la industria textil sea uno de los mayores consumidores de agua. El teñido textil húmedo convencional es un proceso ineficaz, para el que se estima un consumo de 200.000 toneladas de tinte sin usar, que son emitidas en las aguas residuales de la industria textil cada año [23].

La gran mayoría de las plantas de tratamiento de aguas residuales no están equipadas para hacer frente a estos efluentes con contaminantes resistentes; asimismo, la variación en la composición química del efluente para diferentes plantas de teñido es un aspecto que provoca que una gran parte de estos colorantes escape hacia el medio ambiente.

Otros contaminantes orgánicos que pueden estar presentes en el agua son los pesticidas [24], aceites [25], plaguicidas [26], algunos compuestos fenólicos [27, 28], detergentes, fármacos y algunos otros como partículas de aceite/grasa [29, 30], aceite lubricante y diésel [31].

\section{CALIDAD DEL AGUA}

El índice de calidad del agua (ICA) permite estimar el grado de pureza o contaminación de una muestra o un efluente; entre los parámetros que se emplean para estimar el ICA se encuentran: la temperatura, el pH, el color, la conductividad eléctrica, el contenido de sólidos, la DQO (demanda química de oxígeno) y la DBO (demanda bioquímica de oxígeno); que una variación en alguno de estos parámetros indica el grado de contaminación de una muestra de agua. Los colorantes empleados en el proceso de teñido y que se mantienen presentes en las aguas residuales provocan considerables variaciones en las características del agua particularmente en el $\mathrm{pH}$, el color y la concentración de en la DQO [23 y 32].

Sin embargo, los parámetros del ICA estiman en forma global varios contaminantes orgánicos en forma conjunta, por lo que se deben hacer determinaciones particulares y específicas, para distinguir la concentración individual de los compuestos de interés. 


\section{ADSORCIÓN}

De las tecnologías disponibles para reducir la presencia de colorantes y metales pesados en agua y que ha mostrado aceptación es la adsorción, que gana terreno dentro de las técnicas de tratamiento de efluentes textiles por su eficiencia en la eliminación de colorantes, principalmente para aquellos compuestos estables que no pueden removerse o eliminarse por métodos convencionales.

La instalación y puesta en marcha de la mayoría de los procesos de tratamiento requiere de una elevada inversión económica, y resulta complicado, y en algunos de los casos es imposible, adquirir nueva tecnología que permita el tratamiento aguas residuales. Un adecuado proceso de remoción de contaminantes se realiza a través de la adsorción, dando como resultado un efluente de mejor calidad y es un proceso económicamente factible, principalmente para las pequeñas y medianas industrias.

La adsorción es, de entre diversos métodos, uno de los popularmente aceptados por su versatilidad, conveniencia, sencillez, siendo el carbono activado el adsorbente "ideal" para eliminar diversos contaminantes presentes en agua. Por la alta capacidad de adsorción de materia orgánica, el carbono activado es el material adsorbente mayormente utilizado, por lo que es considerado un adsorbente convencional.

Sin embargo, el alto costo de producción del carbono activado limita su aplicación en el tratamiento de aguas residuales; asimismo, la fuerte retención que hace dificulta su regeneración; estos son algunos de los aspectos que impiden su aplicación a gran escala [33]. Por ello, existe una creciente necesidad en la búsqueda de adsorbentes de bajo costo, renovables, preferentemente naturales y disponibles localmente para la remoción de los colorantes.

La adsorción es un proceso físico que se produce por interacciones débiles de largo alcance (fuerzas de van der Waals), lo que permite que partículas, moléculas, o iones queden atrapados o retenidos sobre la superficie de un material. La sustancia que se adsorbe se llama adsorbato y el material que se emplea para su adsorción es el adsorbente o sustrato. Dependiendo de las fuerzas de interacción entre el adsorbato y el adsorbente, se define el tipo de proceso de adsorción. En los procesos de remoción se busca evitar que las moléculas se transformen o degraden, por ruptura o por intercambio de grupos funcionales, evitando la generación de compuestos más reactivos y tóxicos que el compuesto original [34].

La adsorción química o quimisorción se produce por la formación de enlaces (iónico o covalente), por lo que durante la quimisorción la superficie del adsorbente se modifica, dando lugar a un nuevo adsorbente. 


\subsection{Naturaleza química del adsorbato}

Los factores que favorecen o afectan la adsorción del adsorbato son: su tamaño (volumen, masa o peso molecular), los grupos funcionales presentes en su estructura química, la solubilidad, la relación hidrofobicidad / hidrofilicidad, la afinidad relativa entre la solución y el adsorbente, su reactividad y, en el caso de los contaminantes orgánicos, la constante de disociación también afecta a la adsorción [35].

\subsection{Propiedades del adsorbente}

El proceso de adsorción se afecta significativamente por diversos aspectos y propiedades del adsorbente como son la densidad de las partículas del material (tamaño de partícula), el área de superficial, la porosidad, las propiedades físico-químicas de la superficie del adsorbente, la presencia o ausencia de grupos funcionales y la composición elemental, las propiedades térmicas y el pH de la superficie del material [36].

Los adsorbentes no-convencionales suficientemente porosos permiten atrapar o retener moléculas, átomos o iones en su interior. La estructura química de los tejidos vegetales (tallos, hojas, cáscaras, semillas, raíces, etc.) presenta cavidades y poros que permiten el atrapamiento de moléculas pequeñas, como los metales, hasta moléculas complejas como lo son los colorantes. La naturaleza química de estas estructuras poliméricas permite favorables interacciones entre los pares de electrones libres de las especies (adsorbente y adsorbato), por lo que están siendo sumamente atractivos los materiales naturales para ser aplicados en la remoción de contaminantes en agua [37].

\section{ADSORBENTES}

Dentro de las características fundamentales que distinguen a un buen adsorbente están su alta porosidad, su gran superficie de contacto y que presente sitios específicos de adsorción. La mayoría de los adsorbentes que se han utilizado en el control de la contaminación tienen estructura porosa, lo que aumenta el área superficial y, en consecuencia, la velocidad de adsorción. Un buen adsorbente será aquel que presente área superficial suficientemente grande y que requiera menor tiempo para alcanzar el equilibrio de adsorción, por lo que generalmente se prefieren para la eliminación de contaminantes adsorbentes con gran área superficial y alta velocidad de adsorción [38].

\subsection{Adsorbentes convencionales}

Principalmente constituidos por carbono activado, alúmina activada, arenas y zeolitas. Estos adsorbentes son previamente tratados y, una vez utilizados, deben regenerarse para recuperar sus propiedades [39]. Estos materiales actúan como adsorbentes porque pueden formar interacciones intermoleculares débiles con el adsorbato (contaminante); 
las interacciones son fuerzas de atracción electrostáticas o de tipo van der Waals, que conducen a una fisisorción sobre la superficie del adsorbente [40]. Los altos costos de estos materiales limitan su aplicación o son incosteables en un tratamiento para países de escasos recursos.

\subsection{Adsorbentes no-convencionales}

Diversos estudios demuestran la posibilidad de desarrollar materiales con adecuadas capacidades de adsorción a partir fuentes naturales, despertando el interés para explorar estos materiales alternativos considerados "no-convencionales", que permitan reducir o eliminar los contaminantes de naturaleza metálica u orgánica, contenidos en aguas residuales [41-43]. Los adsorbentes no-covencionales (verdes o bioadsorbentes) surgen del aprovechamiento de los materiales de desecho que provienen de la industria alimentaria y agrícola; estos son desechos de fruta, residuos vegetales y plantas.

México y aquellos países que cuentan con una enorme biodiversidad de especies vegetales y frutales son atractivos para el establecimiento de industrias alimentarias o agroindustrias, las cuales generan miles de toneladas de residuos vegetales o frutales por año [44 y 45]. Por ello la utilización de estos residuos, principalmente las cáscaras de las frutas, para la eliminación de los contaminantes presentes en los efluentes acuosos, es un alternativa de gran potencial y de aprovechamiento que presenta diversas ventajas como ser materiales bioadsorbentes de bajo costo, disponibilidad, adecuada capacidad de adsorción, factibilidad de mejorar a través de modificación química, susceptible a la regeneración, degradabilidad lo que conduce a un menor número de problemas en la disposición final posterior a la adsorción [42, 46-49].

A continuación, se muestra una sencilla clasificación, considerando el origen o fuente de obtención de los adsorbentes no convencionales empleados en los métodos de tratamiento de aguas residuales contaminadas [46].

a. biomasa viva y no viva (microorganismos)

b. los materiales de desecho agroindustriales

c. los materiales de desecho de industria alimentaria.

\section{Materiales naturales}

Las pectinas son polisacáridos de origen vegetal, heterogéneos, higroscópicos y solubles en ácidos y agua; presentan propiedades de gelificantes, son estabilizadoras de emulsiones y aportan fibra en la dieta [50]. Se encuentran en la pared celular primaria de frutas y vegetales [51 y 52]. 
Estructuralmente, las pectinas están constituidas por unidades de ácido galacturónico (AG) a través de uniones $\alpha-1,4$ (figura 2) que generan grandes redes enrolladas a través de interacción de puente de hidrógeno entre los hidroxilos no esterificados [53]. Las propiedades funcionales de las pectinas dependen del grado de esterificación y de los grupos funcionales que interrumpan los residuos de AG [54].

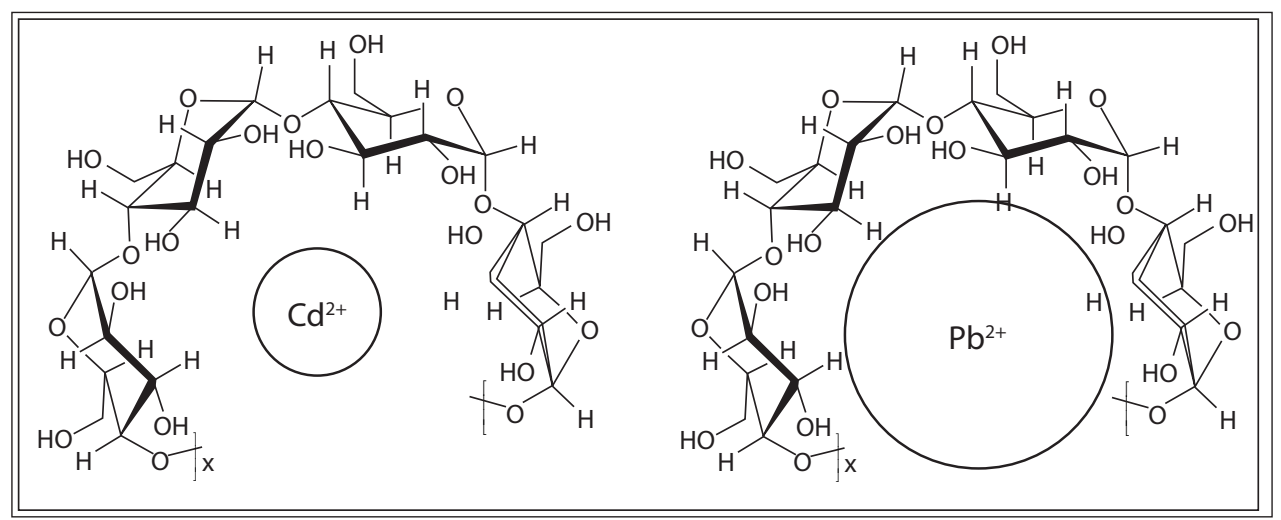

Figura 2. Adsorción de metales pesados por fragmentos de biopolímero a través de interacciones entre los hidroxilos y los iones metálicos.

Fuente: elaboración propia.

Se estima que el consumo anual de pectina en el mundo es de aproximadamente 45 millones de kilogramos. Actualmente, se extrae pectina de una gran cantidad de fuentes como tejocote, uvas, remolacha, manzana y cáscara de cítricos [55]. Por su alto consumo, la obtención a partir de recursos bióticos propios de una región o incluso de residuos agroindustriales es un tema de amplia importancia alrededor del mundo, y tiene como finalidad el aprovechamiento y revalorización de materiales de desecho para menguar el impacto ambiental que estos residuos ocasionan [56].

Tabla 1. Estudios con adsorbentes naturales para la remoción de contaminantes en medio acuoso

\begin{tabular}{|l|c|c|c|c|}
\hline $\begin{array}{c}\text { Adsorbente } \\
\text { Nombre cientifico } \\
\text { (Nombre común) }\end{array}$ & $\begin{array}{c}\text { Tejido } \\
\text { empleado }\end{array}$ & Adsorbato & $\begin{array}{c}\text { Adsorción } \\
\text { máxima } \\
(\mathrm{mg} / \mathrm{g})\end{array}$ & Referencia \\
\hline Allium cepa (Cebolla) & $\mathrm{DF}$ & $\mathrm{Cu}(\mathrm{II})$ & 76.90 & {$[57]$} \\
\cline { 2 - 5 } & $\mathrm{DF}$ & $\mathrm{AM}$ & 1055 & {$[58]$} \\
\hline Allium sativum (Ajo) & $\mathrm{F}$ & $\mathrm{Cu}(\mathrm{II})$ & 66.70 & {$[57]$} \\
\hline Artocarpus camansi (Árbol del pan) & $\mathrm{CF}$ & $\mathrm{AM}$ & 409.00 & {$[59]$} \\
\hline $\begin{array}{l}\text { Benincasa hispida } \\
\text { (Calabaza china) }\end{array}$ & $\mathrm{CF}$ & $\mathrm{Cr}(\mathrm{VI})$ & 18.70 & {$[60]$} \\
\hline
\end{tabular}




\begin{tabular}{|c|c|c|c|c|}
\hline $\begin{array}{c}\text { Adsorbente } \\
\text { Nombre cientifico } \\
\text { (Nombre común) }\end{array}$ & $\begin{array}{l}\text { Tejido } \\
\text { empleado }\end{array}$ & Adsorbato & $\begin{array}{l}\text { Adsorción } \\
\text { máxima } \\
(\mathrm{mg} / \mathrm{g})\end{array}$ & Referencia \\
\hline Mangifera indica (Mango) & $\mathrm{CF}$ & $\begin{array}{l}\mathrm{Cd}(\mathrm{II}) \\
\mathrm{Pb}(\mathrm{II})\end{array}$ & $\begin{array}{l}68.92 \\
99.05\end{array}$ & {$[61]$} \\
\hline Citrus sinensis (Naranja) & $\mathrm{CF}$ & $\begin{array}{c}\text { RN107 } \\
\text { RGBF-2R } \\
\text { RTQ-G125 }\end{array}$ & $\begin{array}{l}1.90 \\
1.70 \\
0.90\end{array}$ & {$[62]$} \\
\hline $\begin{array}{l}\text { Cucumis sativus } \\
\text { (Pepino) }\end{array}$ & $\mathrm{CF}$ & $\begin{array}{c}\mathrm{CV} \\
\mathrm{RDM}\end{array}$ & $\begin{array}{l}33.22 \\
40.82\end{array}$ & [63] \\
\hline $\begin{array}{l}\text { Garcinia mangostana } \\
\text { Mangostino }\end{array}$ & $\mathrm{CF}$ & $\begin{array}{l}\mathrm{Cd}(\mathrm{II}) \\
\mathrm{Pb}(\mathrm{II})\end{array}$ & $\begin{array}{l}68.92 \\
99.05\end{array}$ & [61] \\
\hline $\begin{array}{l}\text { Hylocereus costaricensis } \\
\text { Pitahaya }\end{array}$ & $\mathrm{CF}$ & $\mathrm{AM}$ & 640.00 & [64] \\
\hline \multirow{2}{*}{$\begin{array}{l}\text { Musa paradisiaca } \\
\text { Plátano }\end{array}$} & \multirow{2}{*}{$\mathrm{CF}$} & NAI & 250.00 & {$[65]$} \\
\hline & & $\mathrm{Cu}(\mathrm{II})$ & 70.92 & [66] \\
\hline Cereus hildmannianus (Cáctus) & $\mathrm{CF}$ & ASB & 360.50 & [67] \\
\hline $\begin{array}{l}\text { Punica granatum } \\
\text { (Granada) }\end{array}$ & $\mathrm{P}$ & $\mathrm{AM}$ & 36.36 & $\begin{array}{l}{[68]} \\
{[69]}\end{array}$ \\
\hline $\begin{array}{l}\text { Prunus amygdalus } \\
\text { (almendra) }\end{array}$ & S & NM & 41.34 & [70] \\
\hline $\begin{array}{l}\text { Emblica officinalis } \\
\text { (Grosella de la India) }\end{array}$ & $\mathrm{CF}$ & $\mathrm{Cu}(\mathrm{II})$ & 24.00 & [71] \\
\hline $\begin{array}{l}\text { Simarouba glauca } \\
\text { (aceituna) }\end{array}$ & S & $\mathrm{AM}$ & 111.00 & [72] \\
\hline $\begin{array}{l}\text { Solanum tuberosum } \\
\text { (Papa) }\end{array}$ & $\mathrm{CF}$ & $\mathrm{Cr}(\mathrm{VI})$ & 3.28 & [73] \\
\hline $\begin{array}{l}\text { Zalacca edulis } \\
\text { Salacca }\end{array}$ & $\mathrm{CF}$ & $\mathrm{Cu}(\mathrm{II})$ & $27.03 \mathrm{mg} / \mathrm{g}$ & [74] \\
\hline
\end{tabular}

$\mathrm{DF}=$ dermis de fruto; $\mathrm{F}=$ fruto; $\mathrm{CF}=$ cáscara de fruto; $\mathrm{P}=$ pulpa; $\mathrm{S}=$ semillas; $\mathrm{Cu}(\mathrm{II})=\mathrm{Cobre}(\mathrm{II})$; $\mathrm{AM}=$ azul de metileno; $\mathrm{Cr}(\mathrm{VI})=$ Cromo (VI); Cd(II) = Cadmio (II); Pb(II) = Plomo (II); RN107 = Reactivo Naranja 107; RGBF-2R = Reactivo gris BF-2R; RT Q-G125 = Reactivo turquesa Q-G125; CV $=$ Cristal violeta; $\mathrm{RDM}=$ Rodamina; $\mathrm{NAI}=$ negro ácido $\mathrm{I} ; \mathrm{ASB}=$ Albumina de Suero Bovino, $\mathrm{NM}=$ Naranja de metilo.

Fuente: elaboración propia

A partir de la tabla 1, se puede concluir que los contaminantes metálicos $\mathrm{Pb}^{2+}$, $\mathrm{Cu}^{2+}, \mathrm{Cd}^{2+}$, y el tinte azul de metileno (MB) se eliminan de manera efectiva por piel de naranja $(\mathrm{OP})$, cáscara de plátano $(\mathrm{BP})$, cáscara de granada (PP). La cáscara de ajo (GP) se utiliza de manera efectiva para la eliminación de contaminantes (metales, tintes, y los contaminantes orgánicos) de las aguas residuales dependiendo el proceso de limpieza que se desee dar a un efluente contaminado. Algunos 
ejemplos de cómo pueden ser adaptados los adsorbentes naturales al tratamiento de aguas residuales se muestra en la figura 3 .

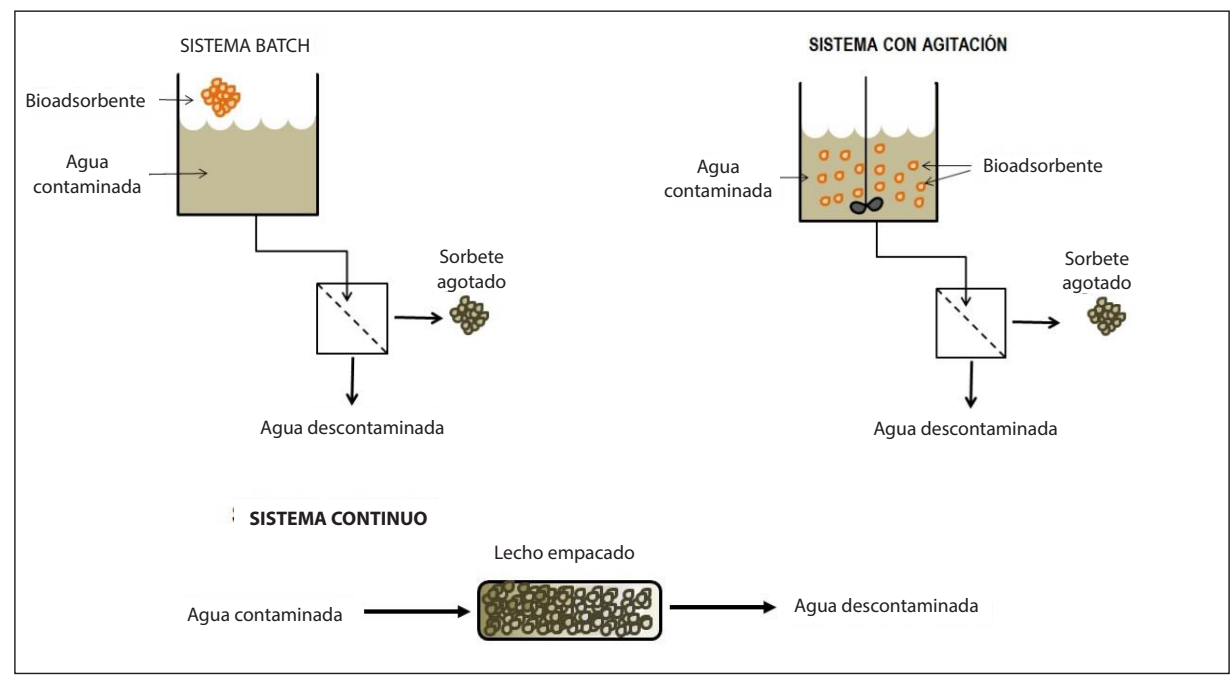

Nota: en el caso del sistema continuo es necesario regenerar o sustituir el lecho empacado.

Figura 3. Representación esquemática de tres sistemas para la decontaminación de agua residual empleando bioadsorbentes.

Fuente: elaboración propia

\section{CONCLUSIONES}

La aplicación de tejidos naturales como materiales no convencionales para la adsorción y remoción de metales y colorantes en el tratamiento de aguas residuales se encuentra actualmente en etapas iniciales. A pesar de que existen varios reportes que mencionan la capacidad adsorptiva de los tejidos de especies vegetales como semillas, troncos, hojas, tallos, etc., se requiere de investigaciones que exploren las aplicaciones del proceso, y de diseño tecnológico acorde con el problema que se necesite atacar.

Es interesante notar que las propiedades y características de la superficie del material natural empleado como adsorbente dependen de varios aspectos como son: (a) procedencia o tipo de fuente; (b) temporada de adquisición o cosecha; (c) estado de la fuente (maduración); (d) calidad, y (e) tratamiento previo que se haga o no al material. Sin embargo, los reportes que incluyen el tratamiento previo realizado a los materiales naturales aumenta el rendimiento de adsorción, aunque esto produciría un incremento en costo del proceso al nivel industrial.

Hasta la fecha, está demostrado que los materiales naturales no convencionales permiten remover eficientemente de agua contaminada: metales pesados, colorantes y 
algunos compuestos orgánicos, abriendo una ventana de exploración para remoción de contaminantes inorgánicos y de nuevas fuentes materiales naturales a partir de otras especies vegetales. Asimismo, la búsqueda de materiales naturales con aplicación como adsorbentes para la remoción de diferentes tipos de contaminantes, a la vez que estén presentes en un mismo efluente, es un tema que tiene vigencia, y día con día recobra mayor interés, por la importancia que tiene el reducir o eliminar por completo las sustancias tóxicas no deseadas en las aguas residuales [12 y 13].

México y muchos países de Latinoamérica cuentan con una amplia gama de especies vegetales que resultan interesantes para ser estudiada su actividad como bioadsorbentes no convencionales en la remoción de contaminantes en agua, desarrollando al mismo tiempo la ingeniería de su aplicación. Se sugiere reorientar los esfuerzos de estudio principalmente sobre aquellas especies de plantas que se consideran malezas, las que son de crecimiento por temporada de lluvia, aquellas que no exhiben uso aplicación terapéutica medicinal o, bien, que no están registras en la medicina tradicional del país de origen.

\section{PERSPECTIVAS}

En la mayoría de los estudios mencionados en los cuales se han empleado adsorbentes naturales, se han realizado en el lote (batch) estático y agitado, por lo que se requieren más estudios orientados al comportamiento del sistema en columna empleando flujos continuos o semi-continuos [75]. Esto ayudará en el diseño de aplicaciones comerciales, lo que conduce a un área de oportunidad para la ingeniería, la química, la mecánica y la eléctrica.

Las propiedades y las características de la superficie del material tienen una importante relación en la consistencia en los resultados, por lo que es necesario caracterizar el material teniendo en cuenta tanto sus propiedades como algunos otros aspectos particulares de cada material que favorezcan la reproducibilidad de los datos experimentales para su adecuado escalamiento y un mejor diseño y desarrollo de tecnología.

La adsorción es un fenómeno físico que depende de varios aspectos. Hasta ahora el establecimiento de un mecanismo exacto del sistema de adsorción para adsorbentes no convencionales ha sido complicado y complejo en muchas de las investigaciones. Solo unos pocos estudios han comentado y propuesto el mecanismo de adsorción que les resulta el más probable, por lo que el mecanismo de adsorción para adsorbentes no convencionales continúa siendo un reto que se debe investigar.

Es conveniente continuar con los estudios al nivel laboratorio y de aplicación de materiales naturales no convencionales para la remoción de contaminantes inorgánicos

Revista Ingenierías Universidad de Medellín, vol. 16, No. 31 pp. 55-73 ISSN 1692-3324 julio-diciembre de 2017/272 p. Medellín, Colombia 
y gaseosos tales como amoníaco, metano, $\mathrm{CO}_{2}$, y $\mathrm{NOx}$ [76 y 77], así como para los contaminantes emergentes. Entre mayor conocimiento se genere, mayores serán las alternativas de solución o tratamiento con un enfoque integral.

\section{REFERENCIAS}

[1] M. F. Chaplin. "Water: its importance to life". Biochem \& Mol Biol Edu., vol. 29, n. ${ }^{\circ}$ 2, pp. 54-59, February 2001

[2] R. Breslow. "The principles of and reasons for using water as a solvent for green chemistry. In Handbook of Green Chemistry. P. T. Anastas Editor. John Wiley and Sosn. Inc. pp. 1-30, March 2010.

[3] J. Carabias, y R. Landa. "Agua, medio ambiente y sociedad. Hacia la gestión integral de los recursos hídricos en México". México, D. F., Ed. UNAM, Colegio de México. Fundación Gonzalo Río Arronte. 2005.

[4] N. Yildiz, R. Gonulsena, H. Koyuncu, A. Calimi. "Adsorption of benzoic acid and hydroquinone by organically modified bentonites". Colloids Surf A, 260, 87-94, June 2005.

[5] L-G. Yan, J. Wang, H-Q. Yu, Q. Wei, B. Du, X-Q. Shan. “Adsorption of benzoic acid by CTAB exchanged montmorillonite". Appl Clay Sci, 37 (3-4), 226-230, January 2007.

[6] M. J. Gil, A. M. Soto, J. I. Usma, O. D. Gutiérrez. "Contaminantes emergentes en aguas, efectos y posibles tratamientos". Producción + Limpia, vol. 7, n. ${ }^{\circ}$, pp. 52-73, julio-diciembre 2012.

[7] R. Vallejo-Rodríguez, M. A. Murillo-Tovar, L. Hernández-Mena, H. Saldarriaga-Noreña, A. López-López. “Compuestos emergentes: Implementación de métodos analíticos para extraer y cuantificar $17 \beta$-estradio, $17 \beta$-etinilestradiol, ibuprofeno y naproxeno en agua. Tecnología y Ciencias del Agua, vol. III, pp. 101-110, febrero-marzo 2012.

[8] B. C. Pan, Y. Xiong, A. M. Li, J. L. Chen, Q. X. Zhang, X. Y. Jin. "Adsorption of aromatic acids on an aminated hypercrosslinked macroporous polymer". React. Funct. Polym, vol. 53, n. ${ }^{\circ}$ 2-3, pp. 63-72, december 2002.

[9] O. A. Titi and O. S. Bello. "An overview of low cost adsorbents for Copper (II) ions removal". J. Biotechnol. \& Biomater, vol. 5, n. ${ }^{\circ}$ 1, pp 1-13, February 2015.

[10] N. Das, R. Vimala, P. Karthika. "Biosorption of heavy metals-An overview". Indian journal of Biotechnology, vol. 7, n. ${ }^{\circ}$ 2, pp. 159-169. 2008.

[11] E. S. Z. El-Ashtoukhy, N. K. Amina, O. Abdelwahab. "Removal of lead (II) and copper (II) from aqueous solution using pomegranate peel as a new adsorbent”. Desalination, vol. 223, n. ${ }^{\circ}$ 1, pp. 162-173, February 2008. 
[12] K. S. W. Sing. "Characterization of porous materials: past, present and future”. Colloids Surf A: Physicochem. Eng. Asp., vol. 241, no. 1-3, pp. 3-7, July 2004.

[13] C. Nava-Ruiz, Y M. Méndez-Armenta. "Efectos neurotóxicos de metales pesados (cadmio, plomo, arsénico y talio)”. Arch. Neurocienc., vol. 16, no. 3, pp. 140-147, julio-septiembre 2011.

[14] A. Mittal, L. Kurup, J. Mittal. "Freundlich and Langmuir adsorption isotherms and kinetics for the removal tartrazine from aqueous solutions using hen feathers". J Hazard Mater, vol. 146, n, ${ }^{\circ} 1-2$, pp. 243-248, December 2007.

[15] T. Robinson, B. Chandran, P. Nigam. "Removal of dyes from a synthetic textile dye effluent by biosorption on Apple pomace and wheat Straw". Water Res, vol. 36, n. ${ }^{\circ}$ 11, pp. 2824-2830, June 2002.

[16] N. Kannan, M. M. Sundaram. "Kinetics and mechanism of removal of methylene blue by adsorption on various carbons. A comparative study". Dyes Pigments, vol. 51, n. ${ }^{\circ}$, pp. 25-40, September 2001.

[17] V. K. Gupta and Suhas. "Application of low-cost adsorbents for dye removal -A review“. $J$ Environ Manage, vol. 90, n. ${ }^{\circ}$ 8, pp. 2312-2342, June 2009.

[18] G. E. Walsh, L. H. Bahner and W. B. Horning. "Toxicity of textile mill effluents to freshwater and estuarine algae, crustaceans and fishes". Environ. Pollut. A, vol. 21, n. ${ }^{\circ}$ 3, pp. 169-179, March 1980.

[19] B. de Campos and M. A. Marin. "Azo dyes: Characterization and toxicity -A review-". TLIST, vol. 2, n. ${ }^{\circ}$ 2, pp. 85-103, April 2013.

[20] A. E. Ghaly, R. Ananthashankar, M. Alhattab and V. V. Ramakrishnan. "Production, characterization and treatment of textile effluents: A critical review". J Chem Eng Process Technol., vol. 5, n. ${ }^{\circ}$ 1, pp. 1-19, January 2014.

[21] F. M. Drumond Chequer, G. A. Rodrigues, E. R. Anastácio, J. Carvalho, M. V. Boldrin and D. Palma. "Textile dyes: dyeing process and environmental impact". In Eco-friendly textile dyeing and finishing, Dr. Melih Gunay (Ed.), InTech, DOI: 10.5772/53659, pp. 151-176, January 2013.

[22] Y. L. Pang and A. Z. Abdullah. "Current status of textile industry wastewater management and research progress in Malaysia: A review". CLEAN-Soil, Air, Water, vol. 41, n. ${ }^{\circ}$, pp. 751-764, August 2013.

[23] A. Hasanbeigi and L. Price. "A technical review of emerging technologies for energy and water efficiency and pollution reduction in the textile industry". J Clean Prod, vol. 95, pp. 30-44, May 2015.

[24] Y. Fushiwaki, K. Uranob. "Adsorption of pesticides and their biodegraded products on clay minerals and soils". J Health Sci, vol. 47, n. ${ }^{\circ}$ 4, pp. 429-432, July 2001.

[25] J. Rubio, F. Tessele. "Removal of heavy metal ions by adsorptive particulate flotation". Miner Eng, vol. 10, n. ${ }^{\circ}$, pp. 671-679, July 1997. 
[26] C. R. Silva, T. F. Gomes, G. C. R. M. Andrade, S. H. Monteiro, A. C. R. Dias E. G. Zagatto, V. L. Tornisielo. "Banana peel as an adsorbent for removing atrazine and ametryne from waters". J Agric Food Chem, vol. 61, n. ${ }^{\circ}$ 10, pp. 2358-2363, January 2013.

[27] S. E. Agarry, C. N. Owabor, A. O. Ajani. "Modified plantain peel as cellulose-based low-cost adsorbent for the removal of 2, 6-dichlorophenol from aqueous solution: adsorption isotherms, kinetic modeling, and thermodynamic studies". Chem Eng Commun, vol. 200, n. ${ }^{\circ}$ 8, pp. 11211147, March 2013.

[28] T. J. I. Edison, M. G. Sethuraman. "Biogenic robust synthesis of silver nanoparticles using Punica granatum peel and its application as a green catalyst for the reduction of an anthropogenic pollutant 4-nitrophenol”. Spectrochim Acta A Mol Biomol Spectrosc, vol. 104, pp. 262-264, March 2013.

[29] U. A. El-Nafaty, I. M. Muhammad, S. Abdulsalam. "Biosorption and kinetic studies on oil removal from produced water using banana peel". Civil Environ Res, vol. 3, n. ${ }^{\circ}$ 7, pp. 125-136, July 2013.

[30] A. Borhan, P. K. Hoong, M. F. Taha. "Biosorption of heavy metal ions, oil and grease from industrial waste water by banana peel”. Appl Mech Mater, vol. 625, pp. 749-752, September 2014.

[31] J. Zou, X. Liu, W. Chai, X. Zhang, B. Li, Y. Wanga, and Y. Ma. "Sorption of oil from simulated seawater by fatty acid modified pomelo peel”. Desalin Water Treat, vol. 339, n. ${ }^{\circ}$, pp. 1-8, July 2014.

[32] A. K. Mehari, S. Gebremedhin and B. Ayele. "Effects of Bahir Dar textile factory effluents on the water quality of the head waters of Blue Nile River, Ethiopia". Int. J Anal Chem., vol. 2015, Article ID 905247, pp. 1-7, November 2015.

[33] G. McDougall. "The physical nature and manufacture of activated carbon”. J S. Afr. Ins. Min. Metall, vol. 91, n. ${ }^{\circ}$, pp. 109-120, April 1991.

[34] R. Sivaraj, C. Namasivayam and K. Kadirvelu. "Orange peel as an adsorbent in the removal of acid violet 17 (acid dye) from aqueous solutions. Waste Management, vol. 21, n. ${ }^{\circ}$, pp. 105-110, January 2001

[35] M. Fomina and G. M. Gadd. "Biosorption: current perspectives on concept, definition and application”. Bioresour. Technol., vol. 160, pp. 3-14, May 2014.

[36] P. A. M. Mourao, O. J. M. Carrot and M. M. L. Ribeiro Carrot. "Application of different equations to adsorption isotherms of phenolic compounds on activated carbons prepared from cork". Carbon, vol. 44, n. ${ }^{\circ}$ 12, pp. 2422-2429, October 2006.

[37] S. Nethaji, A. Sivasamy and A. B. Mandal. "Adsorption isotherms, kinetics and mechanism for the adsorption of cationic and anionic dyes onto carbonaceous particles prepared from Juglans regia shell biomass". Int. J. Environ. Sci. Technol., vol., 10, n. ${ }^{\circ}$ 2, pp. 231-242, March 2013.

[38] B. H. Hameed and M. I. El-Khaiary. "Malachite green adsorption by rattan sawdust: Isotherm, kinetic and mechanism modelling" J Hazard Mater., vol. 159, n. ${ }^{\circ} 2-3$, pp. 574-579, February 2008 . 
[39] F. N. Memon and S. Memon. "Sorption and desorption of basic dyes from industrial wastewater using calix[4]arene based impregnated material” Separation Sci and Technol., vol. 50, n. ${ }^{\circ}$, pp. 1135-1146, January 2015.

[40] J. Tao and A. M. Rappe. "Physical Adsorption: Theory of van der Waals Interactions between particles and clean surfaces". Physic. Rev. Lett., vol. 112, n. ${ }^{\circ}$ 10, pp. 106101, March 2014.

[41] P. D. Pathak, S. A. Mandavgane and B. D. Kulkarni. "Characterizing fruit and vegetable peels as bioadsorbents". Curr Sci., vol. 110, n. ${ }^{\circ}$ 11, pp. 2114-2123, June 2016.

[42] S. Patel. "Potential of fruit and vegetable wastes as novel biosorbents: summarizing the recent studies". Rev Environ Sci Bio/Technol, vol. 11, n. ${ }^{\circ}$ 4, pp. 365-380, December 2012.

[43] A. Bhatnagar, V. J. Vilar, C. M. Botelho and R. A. Boaventura. "Coconut-based biosorbents for water treatment-a review of the recent literatura". Adv Colloid Interface Sci, vol. 160, n. ${ }^{\circ}$ 1-2, pp. 1-15, October 2010.

[44] H. R. Losada, L. L. Rodríguez, J. C. Zorrilla and J. M. Vargas. "The use of organic waste from animals and plants as important input to urban agriculture in México City". Int J Appl Sci Technol, vol. 5, n. ${ }^{\circ}$ 1, pp. 38-44, February 2015.

[45] H. Losada, J. Cortes, J. Rivera and J. Vargas. "Recycling of solid wastes in Mexico City in livestock and agricultural production systems as a sustainable alternative". Field Actions Science Reports. The Journal of Field Actions, vol. 5, pp. 1-11, 2011.

[46] P. Sharma, H. Kaur, M. Sharma and V. Sahore. "A review on applicability of naturally available adsorbents for the removal of hazardous dyes from aqueous waste". Environ Monit Assess, vol. 183, n. ${ }^{\circ}$, pp. 151-195, March 2011.

[47] J. Lehmann, J. Gaunt and M. Rondon. "Bio-char sequestration in terrestrial ecosystems - a review”, Mitig. Adapt. Strateg. Glob. Chang., vol. 11, n. ${ }^{\circ}$, pp. 395-419, March 2006.

[48] D. Mohan, A. Sarswat, Y. S. Ok and C. U. Pittman. "Organic and inorganic contaminants removal from water with biochar, a renewable, low cost and sustainable adsorbent - A critical review", Bioresour. Technol., vol. 160, special n. ${ }^{\circ}$, pp. 191-202, May 2014.

[49] D. Woolf, J. E. Amonette, F. A. Street-Perrott, J. Lehmann and S. Joseph. "Sustainable biochar to mitigate global climate change", Nat. Commun., vol. 1, n. ${ }^{\circ}$ 5, pp. 56, July 2010.

[50] A. D. Zapata, C. A. Escobar, S. F. Cavalitto and R. Hours. "Evaluación de la capacidad de solubilización de pectina de cáscara de limón usando protopectinasa-se". Vitae, vol. 16, n. ${ }^{\circ}$ 1, pp. 67-74, Enero 2009.

[51] R. Sthornvit and N. Pitak. "Oxygen permeability and mechanical properties of banana films". Food Res Int., vol. 40, n. ${ }^{\circ}$ 3, pp. 365-370, April 2007.

[52] V. Coma. "Polysaccharide-based biomaterials with antimicrobial and antioxidant properties". Polímeros, vol. 20, n. ${ }^{\circ}$ 2, pp. 1-12, marzo-abril 2010.

[53] M. L. Fishman and P. H. Cooke. "The structure of high-methoxyl sugar acid gels of citrus pectin as determined by AFM". Carbohydr. Res., vol. 344, n. ${ }^{\circ}$ 14, pp. 1792-1797, September 2009 . 
[54] S. Yoo, M. L. Fishman, A. T.Hotchkiss and H. G. Lee. "Viscometric behavior of high-methoxy and low-methoxy pectin solutions". Food Hydrocolloids, vol. 20, n. ${ }^{\circ}$ 1, pp. 62-67, March 2006.

[55] K. K. Woo, Y. Y. Chong, S. K. Li Hiong and P. Y. Tang. "Pectin extraction and characterization from red dragon fruit (Hylocereus polyrhizus): A preliminary study". J Biol. Sci., vol. 10, n. ${ }^{\circ}$ 7, pp. 631-636, October 2010.

[56] H. Ueno, M. Tanak, M. Hosino, M. Sasaki and M. Goto. "Extraction of valuable compounds from the albedo of Citrus junos using subcritical water". Sep. Purif. Technol., vol. 62, n. ${ }^{\circ}$, pp. 513-516, September 2008.

[57] A. Chowdhury, A. Bhowal and S. Datta. "Equilibrium, thermodynamic and kinetic studies for removal of copper (II) from Aqueous solution by onion and garlic skin". Water, vol. 4, pp. 37-51, November 2012.

[58] S. Saber-Samandari and J. Heydaripour. "Onion membrane: an efficient adsorbent for decoloring of wastewater”. J Environ. Health Sci. Eng., vol. 13, n. 16, pp. March 2015.

[59] L. B. L. Lim, N. Priyantha, D. T. B. Tennakoon, H. I. Chieng, M. K. Dahri and M. Suklueng. "Breadnut peel as a highly effective low-cost biosorbent for methylene blue: equilibrium, thermodynamic and kinetic studies". Arabian J Chem, Doi: 10.1016/j.arabjc.2013.12.018, January 2014.

[60] K. M. Sreenivas, M. B. Inarkar, S. V. Gokhale and S. S. Lele. "Re-utilization of ash gourd (Benincasa hispida) peel waste for chromium (VI) biosorption: equilibrium and column studies". J Environ Chem Eng, vol. 2, n. ${ }^{\circ}$ 1, pp. 455-462, March 2014.

[61] M. Iqbal, A. Saeed and S. I. Zafar. "FTIR spectrophotometry, kinetics and adsorption isotherms modeling, ion exchange, and EDX analysis for understanding the mechanism of $\mathrm{Cd}^{2+}$ and $\mathrm{Pb}^{2+}$ removal by mango peel waste". J Hazard Mater, vol. 164, n. ${ }^{\circ}$ 1, pp. 161-171, May 2009.

[62] G. E. Do Nascimento, M. M. Menezes, N. Campos, O. R. Sá Da Rocha and V. Lins Da Silva. "Adsorption of azo dyes using peanut hull and orange peel: a comparative study". Environ Technol, vol. 35, n. ${ }^{\circ} 11$, pp. 1436-1453, 2014.

[63] T. Smitha, T. Santhi, A. L. Prasad and S. Mononmani. "Cucumis sativus used as adsorbent for the removal dyes from aqueous solution". Arabian J Chem., vol. 4, pp. August 2012.

[64] N. Priyantha, L. B. L. Lim and M. K. Dahri. "Dragon fruit skin as a potential biosorbent for the removal of methylene blue dye from aqueous solution”. Int Food Res J., vol. 22, n. ${ }^{\circ}$ 5, pp. 2141-2148, September - October 2015.

[65] C. Palma, E. Contreras, J. Urra and M. J. Martínez. "Eco-friendly technologies based on banana peel use for the decolourization of the dyeing process wastewater". Waste Biomass Valorization, vol. 2, n. ${ }^{\circ}$, pp. 77-86, November 2011.

[66] M. Thirumavalavan, Y. L. Lai, L. C. Lin and J. F. Lee. "Cellulose-based native and surface modified fruit peels for the adsorption of heavy metal ions from aqueous solution Langmuir adsorption isotherms". J Chem Eng Data, vol. 55, n. 3, pp. 1186-1192, October 2010. 
[67] A. Gilioli, M. Cavejon and M. G. N. Quadri. "C. hildmannianus peel for protein adsorption”. Chem Eng Trans, vol. 32, pp. 1099-1104, June 2013

[68] A. Bhatnagar and A. K. Minocha. "Adsorptive removal of 2, 4-dichlorophenol from water utilizing Punica granatum peel waste and stabilization with cement". J Hazard Mater, vol. 168, n. $^{\circ} 2-3$, pp. 1111-1117, September 2009.

[69] F. Güzel, Ö. Aksoy and G. Akkaya. "Application of pomegranate (Punica granatum) pulp as a new biosorbent for the removal of a model basic dye (methylene blue)". World Appl Sci J., vol. 20, n. ${ }^{\circ}$, pp. 965-975, January 2012.

[70] F. Deniz. "Adsorption properties of low-cost biomaterial derived from Prunus amygdalus L. for dye removal from water". The Scientific World Journal, vol. 2013, Article ID 961671, pp. 1-8, June 2013.

[71] R. A. K. Rao and S. Ikram, "Sorption studies of Cu (II) on gooseberry fruit (Emblica officinalis) and its removal from electroplating wastewater". Desalination, vol. 277, n. ${ }^{\circ}$ 1-3, pp. 390-398, August 2011.

[72] B. Jeyagowri and R. T. Yamuna. "Biosorption of Methylene blue from aqueous solutions by modified mesoporous Simarouba glauca seed shell powder". Global NEST J., vol. 17, n. ${ }^{\circ}$, pp. 701-715, November 2015

[73] F. Mutongo, O. Kuipa and P. K. Kuipa. "Removal of $\mathrm{Cr}(\mathrm{VI})$ from aqueous solutions using powder of potato peelings as a low cost sorbent". Bioinorg Chem Appl, vol. 2014, Article ID 973153, pp. 1-7, June 2014.

[74] C. Sirilamduan, C. Umpuch and P. Kaewsarn. "Removal of copper from aqueous solutions by adsorption using modify Zalacca edulis peel modify. Songklanakarin J Sci Technol, vol. 33, n. ${ }^{\circ}$ 6, pp. 725-732, Nov-Dec. 2011

[75] D. Zhou, L. Zhang, J. Zhou and S. Guo. "Development of a fixed-bed column with cellulose/ chitin beads to remove heavy-metal ions". J Appl Polym Sci, vol. 94, n. 2 , pp. 684-691, Aug 2004.

[76] L. Giraldo, M. Bastidas-Barranco and J. C. Moreno-Pirajan. "Preparation of carbon monoliths from orange peel for NOx retention”. Orient J Chem., vol. 30, pp. 1517-1528, 2014.

[77] G. K. Gupta, S. De, A. Franco, A. M. Balu and R. Luque. "Sustainable biomaterials: Current trends, challenges and applications”. Molecules, vol. 21, n. 48, pp. 1-11, January 2016. 